Pacific Journal of Mathematics

CHAIN CONDITIONS AND PURE-EXACTNESS

CHAN CONDITONS AND PURE-BXaCiNES 


\title{
CHAIN CONDITIONS AND PURE-EXACTNESS
}

\author{
M. B. REGE AND K. VARADARAJAN
}

Let $R$ be a ring and $M$ any (right) $R$-module. For any set $I$ let $M^{(I)}$ and $M^{I}$ denote the direct sum and respectively the direct product of copies of $M$ indexed by the set $I$. For any cardinal number $r$, let $\mathscr{C}_{r}$ denote the class of $R$-modules admitting a generating set of cardinality $\leqq r$. In this paper we study the relationship between the pure-exactness of the sequence $0 \rightarrow M^{(I)} \rightarrow M^{I} \rightarrow M^{I} / M^{(I)} \rightarrow 0$ with respect to $\mathscr{C}_{r}$ under the functor Hom $_{R}$ and chain conditions on suitably defined families of $R$-modules. This study led us to the introduction of five properties $A_{r}, A_{(r)}, D_{r}, D_{(r)}$, and $P_{r}$ for any $R$-module $M$. We also study the effect of base extension (both covariant and contravariant) of the ring $R$ on modules having any (or some) of the above mentioned properties. Finally we obtain necessary and sufficient conditions for

$$
0 \longrightarrow \oplus M_{\alpha} \longrightarrow \pi M_{\alpha} \longrightarrow \pi M_{\alpha} / \oplus M_{\alpha} \longrightarrow 0
$$

to be pure-exact with respect to $\mathscr{C}_{k}$, where $\left\{M_{\alpha}\right\}$ is any family of $R$-modules and $k$ any integer $\geqq 1$.

Introduction. Let $\mathscr{E}$ be any family of $R$-modules. An exact sequence $0 \rightarrow M^{\prime} \rightarrow M \stackrel{\varepsilon}{\rightarrow} M^{\prime \prime} \rightarrow 0$ of $R$-modules is said to be pureexact with respect to the family $\mathscr{E}$ (for the functor Hom $=\mathrm{Hom}_{R}$ ) if $\varepsilon_{*}: \operatorname{Hom}(E, M) \rightarrow \operatorname{Hom}\left(E, M^{\prime \prime}\right)$ is onto for all $E \in \mathscr{E}$. Given a module $M$ and a set $I$ let $M^{(I)}$ and $M^{I}$ denote respectively the direct sum and the direct product of copies of the same module $M$ indexed by $I$. In his paper [6] H. Lenzing, among other things, studies equivalent conditions under which the sequence

$$
0 \longrightarrow M^{(I)} \longrightarrow M^{I} \longrightarrow M^{I} / M^{(I)} \longrightarrow 0
$$

is pure-exact with respect to the family of cyclic modules. His result (Proposition 1 of [6]) is a generalization of Carl Faith's result [4] concerning necessary and sufficient conditions for $M^{(I)}$ to be injective. Let $r$ be any cardinal number and $\mathscr{C}_{r}$ the class of $R$-modules admitting a generating set with cardinality $\leqq r$. One of the objectives of the present paper is to study the extent to which Lenzing's results could be generalized to furnish conditions under which (1) will be pure-exact with respect to $\mathscr{C}_{r}$.

Throughout this paper $k$ will denote an integer $\geqq 1$. $R$ will denote a ring with $1, R$-mod and $\bmod -R$ will denote the categories of left resp. right unitary $R$-modules. Unless otherwise mentioned, by an $R$-module we mean an object of $\bmod -R$. All the concepts 
used in this paper are right sided unless otherwise mentioned. When we talk of homomorphisms between rings we always assume that they preserve the identity elements. When $R$ is a subring of $S$ we assume $1_{R}=1_{S}$ so that the inclusion map $j: R \rightarrow S$ is a ring homomorphism. For any set $J$, the cardinality of $J$ will be denoted by $|J|$.

The results of Lenzing in [6] suggest the introduction and study of five properties $A_{r}, A_{(r)}, D_{r}, D_{(r)}$, and $P_{r}$ for any $M \in \bmod -R$ and any cardinal number $r \neq 0$. Let $J$ be any set with $|J|=r$. For any $\underline{x}=\left(x_{j}\right)_{j \in J} \in M^{J}$ and $\underline{\lambda}=\left(\lambda_{j}\right)_{j \in J} \in R^{(J)}$ let $\langle\underline{x}, \underline{\lambda}\rangle=\sum_{j} x_{j} \lambda_{j}$. For any subset $T$ of $M^{J}$ let $L_{T}=\left\{\underline{\lambda} \in R^{(J)} \mid\langle\underline{x}, \underline{\lambda}\rangle=0\right.$ for all $\left.\underline{x} \in T\right\}$. We write $\mathscr{F}_{r}(M)$ (resp. $\mathscr{F}_{(r)}(M)$ ) for the family $\left\{L_{T} \mid T\right.$ subset of $\left.M^{J}\right\}$ (resp. $\left\{L_{T} \mid T\right.$ subset of $\left.\left.M^{(J)}\right\}\right) . \quad M \in \bmod -R$ is said to have the property $A_{r}$ (resp. $A_{(r)}$ ) if $\mathscr{F}_{r}(M)$ (resp. $\mathscr{F}_{(r)}(M)$ ) has the ascending chain condition. Similarly, $M$ is said to have the property $D_{r}$ (resp. $D_{(r)}$ ) if $\mathscr{F}_{r}(M)$ (resp. $\mathscr{F}_{(r)}(M)$ ) has the descending chain condition. When $r=k, A_{k}$ and $A_{(k)}$ are equivalent by definition and we use the common notation $A_{k}$. Similarly $D_{k}$ and $D_{(k)}$ are equivalent and we use the common notation $D_{k}$. It turns out $A_{r}$ and $A_{(r)}$ are equivalent for any cardinal $r$. However, we use either notation depending on the context. $M$ is said to have $P_{r}$ if for any set $I, 0 \rightarrow M^{(I)} \rightarrow$ $M^{I} \rightarrow M^{I} / M^{(I)} \rightarrow 0$ is pure-exact with respect to $\mathscr{C}_{r}$. It also turns out that $A_{k}$ and $P_{k}$ are equivalent, whereas for an infinite cardinal $r$ one has only the implication $A_{r} \Rightarrow P_{r}$. We show by means of an example that the implication $P_{r} \Rightarrow A_{r}$ is not true. It turns out that the properties $A_{r}, D_{r}$, and $D_{(r)}$ are hereditary, in the sense that if $M \in \bmod -R$ has any one of these properties and $N$ is a submodule of $M$, then $N$ also has the same property. Since $A_{k}$ and $P_{k}$ are equivalent, it follows that $P_{k}$ is hereditary. We give an example to show that $P_{r}$ is not hereditary for an infinite cardinal $r$.

Let $R$ be a subring of $S$ and $j: R \rightarrow S$ the inclusion. Using $j$ we regard $S$ (and also $S / R$ ) as an element of $R$-mod. Let $M \in \bmod$ $R$. If $M^{\prime}=M \otimes_{R} S \in \bmod -S$ has property $A_{(r)}$ (resp. $\left.D_{(r)}\right)$ and if $\operatorname{Tor}_{1}^{R}(M, S / R)=0$, then we show that $M \in \bmod -R$ property $A_{(r)}$ (resp. $\left.D_{(r)}\right)$. We say that a ring $R$ has a certain property if $R$ considered as an element of $\bmod -R$ in the usual way has that property. An immediate consequence of the above result is that if $S$ has $A_{(r)}$ (resp. $\left.D_{(r)}\right)$, then $R$ has $A_{(r)}$ (resp. $\left.D_{(r)}\right)$. Also the above results yield an alternative proof of the following well-known result which can be found in Chap. I, $\S 3, \mathrm{n}^{\circ} 5$ of [2]: If $R$ is a subring of a right noetherian (artinian) ring $S$ and if $S / R$ is left flat over $R$, then $R$ is right noetherian (artinian). Also we prove that a regular ring $R$ has property $A_{1}$ if and only if it is semisimple.

Next we consider the situation where there exists a ring homo- 
morphism $f: R \rightarrow S$. Let $M \in \bmod -R$ and $M^{\prime}=\operatorname{Hom}_{R}(S, M) \in \bmod -S$. We study conditions under which $M^{\prime}$ having property $A_{r}$ (resp. $D_{r}$ ) implies $M$ has the same property. We apply these results to derive conditions under which $\Sigma$-injectivity of $M^{\prime}$ implies $\Sigma$-injectivity of $M$.

In [7] B. Sarath and K. Varadarajan obtain necessary and sufficient conditions for the direct sum $\oplus M_{\alpha}$ of a given family $\left\{M_{\alpha}\right\}_{\alpha \in I}$ of modules to be injective. Finally we generalize results in [7] and furnish conditions under which the sequence

$$
0 \longrightarrow \oplus M_{\alpha} \longrightarrow \Pi M_{\alpha} \longrightarrow \Pi M_{\alpha} / \oplus M_{\alpha} \longrightarrow 0
$$

is pure-exact with respect to $\dot{\mathscr{C}}_{k}$, where $\left\{M_{\alpha}\right\}_{\alpha \in I}$ is an arbitrary family in $\bmod -R$.

1. Preliminaries. Let $R$ be a ring, $U \in \bmod -R, W \in R$-mod and $M$ an abelian group. Let $\theta: U \boldsymbol{\bigotimes}_{R} W \rightarrow M$ be a given homomorphism of abelian groups. For any $(u, w) \in U \times W$ we write $\langle u, w\rangle$ for the element $\theta(u \otimes w)$. Given any subset $X$ of $U$ let $L_{X}=\{w \in W \mid\langle u, w\rangle=$ 0 for all $u \in X\}$. Clearly, $L_{X}$ is an additive subgroup of $W$. Let $\mathscr{F}_{W}(\theta)=\left\{L_{X} \mid X\right.$ any subset of $\left.U\right\}$. We remark that $L_{X}$ has been called an annihilator with respect to $\langle$,$\rangle in [1, \S 24]$.

Proposition 1.1. With the above notation, the following conditions are equivalent.

(a) $\mathscr{F}_{W}(\theta)$ satisfies the ascending chain condition.

(b) There do not exist any sequences $\left\{u^{(n)}\right\}_{n \geqq 1},\left\{w^{(n)}\right\}_{n \geqq 1}$ with $u^{(n)} \in U, w^{(n)} \in W$ and satisfying

$\left.\begin{array}{l}\text { (i) }\left\langle u^{(n)}, w^{(n)}\right\rangle \neq 0 \\ \text { (ii) }\left\langle u^{(m)}, w^{(n)}\right\rangle=0\end{array}\right\}$ for all $m>n \geqq 1$.

Proof. $\quad(\mathrm{a}) \Rightarrow(\mathrm{b})$. Suppose there exist sequences $u^{(n)}$ in $U, w^{(n)}$ in $W$ satisfying (i) and (ii) of (b). Let $X_{n}$ be the subset $X_{n}=$ $\left\{u^{(k)} \mid k \geqq n\right\}$ of $U$. Then $L_{X_{n}} \subset L_{X_{n+1}}$. From $\left\langle u^{(n)}, w^{(n)}\right\rangle \neq 0$ and $\left\langle u^{(m)}, w^{(n)}\right\rangle=0$ for $m>n$ we see that $w^{(n)} \in L_{X_{n+1}}-L_{X_{n}}$. Thus $L_{X_{1}} \subset$ $L_{X_{2}} \cdots$ is a strict ascending chain in $\mathscr{F}_{W}(\theta)$ contradicting (a).

(b) $\Rightarrow$ (a). Suppose $L_{1} \subset L_{\Delta_{2}} \subset \cdots$ is a strictly increasing chain of objects in $\mathscr{F}_{W}(\theta)$, where $\Delta_{i}$ are subsets of $U$. Let $w^{(n)} \in L_{\Delta_{n+1}}-$ $L_{n}$. Then there exists a $u^{(n)} \in \Delta_{n}$ with $\left\langle u^{(n)}, w^{(n)}\right\rangle \neq 0$. Since $u^{(m)} \in$ $\Delta_{m}$ and $w^{(n)} \in L_{\Delta_{m}}$ for all $m>n$ we get $\left\langle u^{(m)}, w^{(n)}\right\rangle=0$ for $m>n$. This contradicts (b).

Proposition 1.2. With the same notation as in Proposition 1.1, the following are equivalent.

(a) $\mathscr{F}_{W}(\theta)$ satisfies the descending chain condition. 
(b) There do not exist sequences $\left\{u^{(n)}\right\}_{n \geqq 1},\left\{w^{(n)}\right\}_{n \geqq 1}$ with $u^{(n)} \in U$, $w^{(n)} \in W$ and satisfying

$\left.\begin{array}{l}\text { (i) }\left\langle u^{(n)}, w^{(n)}\right\rangle \neq 0 \\ \text { (ii) }\left\langle u^{(n)}, w^{(m)}\right\rangle=0\end{array}\right\}$ for all $m>n \geqq 1$.

The proof is similar to that of Proposition 1.1 and hence omitted.

For any subset $Y$ of $W$ let $L_{Y}=\{u \in R \mid\langle u, y\rangle=0$ for all $y \in Y\}$. Then $L_{Y}$ is a $Z$-submodule of $U$. Let $\mathscr{F}_{U}(\theta)=\left\{L_{Y} \mid Y\right.$ any subset of $W\}$. The following proposition is an immediate consequence of Propositions 1.1 and 1.2 (cf. Proposition 24.3 of [1]).

Proposition 1.3. The following are equivalent.

(a) $\mathscr{F}_{W}(\theta)$ satisfies the ascending (resp. descending) chain condition.

(b) $\mathscr{F}_{U}(\theta)$ satisfies the descending (resp. ascending) chain condition.

2. The five properties, Let $M \in \bmod -R$ and $J$ a nonempty set. We take $U=M^{J}$ and $W=R^{(J)}$. For any $\underline{x}=\left(x_{j}\right)_{j \in J}$ in $U$ and $\underline{\lambda}=$ $\left(\lambda_{j}\right)_{j \in J}$ in $R^{(J)}$ let $\phi: U x W \rightarrow M$ be given by $\phi(x, \underline{\lambda})=\Sigma x_{j} \lambda_{j}$. Notice that the sum $\Sigma x_{j} \lambda_{j}$ is only a finite sum since $\underline{\lambda} \in R^{(J)}$. The map $\dot{\phi}$ clearly induces a homomorphism $\theta: M^{J} \bigotimes_{R} R^{(J)} \rightarrow M$. Also $R^{(J)}$ is a left, right $R$-bimodule. Hence $M^{J} \otimes_{R} R^{(J)}$ is in a natural way a right $R$-module. It is clear that $\theta$ is an $R$-homomorphism. For any subset $T$ of $U, L_{T}=\{\underline{\lambda} \in W \mid\langle\underline{x}, \underline{\lambda}\rangle=0$ for all $\underline{x} \in T\}$ is an $R$-submodule of $W=R^{(J)}$. In this case the family $\mathscr{F}_{W}(\theta)$ introduced in $\S 1$ will be denoted by $\mathscr{F}^{J}(M)$. If $J$ and $J^{\prime}$ are any two sets with $|J|=\left|J^{\prime}\right|=r \neq 0$, using a bijection of $J$ with $J^{\prime}$ we can get a bijection of $\mathscr{F}^{J}(M)$ with $\mathscr{F}^{J^{\prime}}(M)$ preserving inclusions. Hence we write $\mathscr{F}_{r}(M)$ for any $\mathscr{F}^{J}(M)$ with $|J|=r$.

We can respect the above considerations with $U=M^{(J)}, W=R^{(J)}$, and $\phi(\underline{x}, \underline{\lambda})=\Sigma x_{j} \lambda_{j}$ for any $\underline{x}=\left(x_{j}\right)$ in $M^{(J)}$ and $\underline{\lambda}=\left(\lambda_{j}\right)$ in $R^{(J)}$. In this case we denote the family $\mathscr{F}_{W}(\theta)$ by either $\mathscr{F}^{(J)}(\boldsymbol{M})$ or $\mathscr{F}_{(r)}(\boldsymbol{M})$. Since $R^{(\phi)}=0$, we do not consider the case $r=0$ at all. Thus throughout this paper $r$ denotes a cardinal $\neq 0$.

Definition 2.1. $M$ is said to have the property $A_{r}$ (resp. $A_{(r)}$ ) if $\mathscr{F}_{r}(M)$ (resp. $\mathscr{F}_{(r)}(M)$ ) has the ascending chain condition.

Definition 2.2. $M$ is said to have the property $D_{r}$ (resp. $D_{(r)}$ ) if $\mathscr{F}_{r}(M)$ (resp. $\mathscr{F}_{(r)}(M)$ ) has the descending chain condition.

Definition 2.3. $M$ is said to have the property $P_{r}$ if for every 
set $I, 0 \rightarrow M^{(I)} \rightarrow M^{I} \rightarrow M^{I} / M^{(I)} \rightarrow 0$ is pure-exact with respect to $\mathscr{C}_{r}$.

The following two lemmas are implicit in [5].

Lemma 2.4 (Fieldhouse). Let $B \subset A, N \subset M$ be $R$-modules, $\eta: A \rightarrow$ $A / B$ and $\varepsilon: M \rightarrow M / N$ the canonical quotient maps. Suppose $\varepsilon_{*}$ : $\operatorname{Hom}(A / B, M) \rightarrow \operatorname{Hom}(A / B, M / N)$ is onto. Then for any $f: A \rightarrow M$ satisfying $f(B) \subset N$, there exists a $g: A \rightarrow N$ such that $f / B=g / B$.

LEMMA 2.5 (Fieldhouse). With the same notation as in Lemma 2.4, suppose $\varepsilon_{*}: \operatorname{Hom}(A, M) \rightarrow \operatorname{Hom}(A, M / N)$ is onto. Suppose for any $f: A \rightarrow M$ satisfying $f(B) \subset N$, there exists a $g: A \rightarrow N$ such that $g / B=f / B$. Then $\varepsilon_{*}: \operatorname{Hom}(A / B, M) \rightarrow \operatorname{Hom}(A / B, M / N)$ is onto.

The set of natural numbers will be denoted by $N$. For each cardinal number ${ }^{-} r$ we denote the class of modules isomorphic to $R^{(J)} / L$ with $|J|=r$ and $L$ countably generated submodule of $R^{(J)}$ by $\mathscr{C}_{r}^{\prime}$. In the statement of Propositions 2.6 and $2.7, M$ is a given object in $\bmod -R, r$ a given cardinal number and $J$ a set with $|J|=r$.

Proposition 2.6. Consider the following statements:

(a) $M$ has property $A_{r}$.

(a') $M$ has property $A_{(r)}$.

(b) There do not exist any sequences $\left\{\underline{x}^{(n)}\right\}_{n \geqq 1},\left\{\underline{\lambda}^{(n)}\right\}_{n \geqq 1}$ with $\underline{x}^{(n)} \in$ $M^{J}, \underline{\lambda}^{(n)} \in R^{(J)}$ and satisfying

$\left.\begin{array}{l}\text { (i) }\left\langle\underline{\boldsymbol{x}}^{(n)}, \underline{\boldsymbol{\lambda}}^{(n)}\right\rangle \neq 0 \\ \text { (ii) }\left\langle\boldsymbol{\boldsymbol { x }}^{(m)}, \underline{\lambda}^{(n)}\right\rangle=0\end{array}\right\}$ for $m>n \geqq 1$.

(b') Same as (b) with $M^{(J)}$ replacing $M^{J}$.

(c) For any set $I$, any submodule $L$ of $R^{(J)}$ and any map $f$ : $R^{(J)} \rightarrow M^{I}$ satisfying $f(L) \subset M^{(I)}$ there exists a $g: R^{(J)} \rightarrow M^{(I)}$ such that $g / L=f / L$.

(d) For any set $I$, the sequence

$$
0 \longrightarrow M^{(I)} \longrightarrow M^{I} \longrightarrow M^{I} / M^{(I)} \longrightarrow 0
$$

is pure-exact with respect to $\mathscr{C}_{r}$.

(e) The sequence $0 \rightarrow M^{(N)} \rightarrow M^{N} \rightarrow M^{N} / M^{(N)} \rightarrow 0$ is pure-exact with respect to $\mathscr{C}_{r}^{\prime}$.

In general, we have $(\mathrm{a}) \Leftrightarrow(\mathrm{b}) \Leftrightarrow\left(\mathrm{b}^{\prime}\right) \Leftrightarrow\left(\mathrm{a}^{\prime}\right)$ and $(\mathrm{a}) \Rightarrow(\mathrm{c}) \Rightarrow(\mathrm{d}) \Rightarrow$ (e). If $r$ is a finite cardinal all the statements (a) to (e) are equivalent.

Proof. The equivalences $(a) \Leftrightarrow(b)$ and $\left(a^{\prime}\right) \Leftrightarrow\left(b^{\prime}\right)$ follow from Proposition 1.1. (b) $\Rightarrow\left(\mathrm{b}^{\prime}\right)$ is trivial since $M^{(J)} \subset M^{J}$. 
$\left(\mathrm{b}^{\prime}\right) \Rightarrow(\mathrm{b})$ : For each $n \geqq 1$ let $J_{n}=\left\{j \in J \mid \lambda_{j}^{(i)} \neq 0\right.$ for some $i$ in $1 \leqq i \leqq n\}$. Since $\underline{\lambda}^{(i)} \in R^{(J)}$ we see that each $J_{n}$ is a finite set. Let $\underline{y}^{(n)} \in M^{(J)}$ be defined by $y_{j}^{(n)}=x_{j}^{(n)}$ for $j \in J_{n}$ and $y_{j}^{(n)}=0$ for $j \in J-$ $J_{n}$. Then clearly $\left\langle\underline{y}^{(m)}, \underline{\lambda}^{(n)}\right\rangle=\left\langle\underline{x}^{(m)}, \underline{\lambda}^{(n)}\right\rangle$ for all $m \geqq n \geqq 1$. In particular, $\left\langle\underline{y}^{(n)}, \underline{\lambda}^{(n)}\right\rangle=0,\left\langle\underline{y}^{(m)}, \underline{\lambda}^{(n)}\right\rangle=0$ for $m>n \geqq 1$.

(a) $\Rightarrow$ (c): Let $L$ be a submodule of $R^{(J)}$ and $f: R^{(J)} \rightarrow M^{I}$ be a map satisfying $f(L) \subset M^{(I)}$. Let $\left\{\underline{e}^{j}\right\}$ denote the canonical basis of $R^{(J)}$, namely, $\underline{e}^{j}=\left(u_{j^{\prime}}\right)_{j^{\prime} \in J}$ with $u_{j^{\prime}}=0$ for $j^{\prime} \neq j$ and $u_{j}=1$. Let $f\left(\underline{e}^{j}\right)=\left(x_{i}^{j}\right)_{i \in I}$ with $x_{i}^{j} \in M$. For any $i \in I$ define $\underline{y}^{i} \in M^{J}$ by $y_{j}^{i}=x_{i}^{j}$ $\forall j \in J$. Let $H(I)$ denote the set of finite subsets of $I$. For any $F \in H(I)$ let $K_{F}=\left\{\underline{\lambda} \in R^{(J)} \mid\left\langle\underline{y}^{i}, \underline{\lambda}\right\rangle=0\right.$ for $\left.i \in I-F\right\}$. If $F, F^{\prime}$ in $H(I)$ satisfy $F \subset F^{\prime}$ we have $K_{F} \subset K_{F^{\prime}}$. Each $K_{F}$ is an element of $\mathscr{F}_{r}(M)$. Since $\mathscr{F}_{r}(M)$ satisfies the ascending chain condition, there exists an $F_{0} \in H(I)$ such that $K_{F_{0}}$ is maximal in $\left\{K_{F}\right\}_{F \in H(I)}$. For any $F \in H(I)$ we have $K_{F} \subset K_{F \cup F_{0}} \supset K_{F_{0}}$. The maximality of $K_{F_{0}}$ yields $K_{F \cup F_{0}}=K_{F_{0}}$. Hence $K_{F_{0}} \supset K_{F}$ for all $F \in H(I)$.

Let $\underline{\lambda}=\left(\lambda_{j}\right)_{j \in J}$ be any element of $L$. The $i$ coordinate of $f(\underline{\lambda})$ is $\sum_{j} x_{i}^{j} \lambda_{j}$. Since $f(\underline{\lambda}) \in M^{(I)}$ we see that there exists an $F \in H(I)$ (depending on $\underline{\lambda}$ ) such that $\sum_{j} x_{i}^{j} \lambda_{j}=0$ for $i \in I-F$. Hence $\left\langle\underline{y}^{i}, \underline{\lambda}\right\rangle=$ $\sum_{j} y_{j}^{2} \lambda_{j}=\sum_{j} x_{i}^{j} \lambda_{j}=0$ for $i \in I-F$. This proves that $L \subset \bigcup_{F^{\prime} \in H^{(I)}} K_{F}$. From $K_{F} \subset K_{F_{0}}$ for all $F \in H(I)$ we get $L \subset K_{F_{0}}$. Let $\underline{v}^{j} \in M^{(I)}$ be defined by $v_{i}^{j}=\left\{\begin{array}{l}x_{i}^{j} \text { for } i \in F_{0} \\ 0 \text { for } i \in I-F_{0} .\end{array}\right.$ The map $g: R^{(r)} \rightarrow M^{(I)}$ carrying $e^{j}$ to $\underline{v}^{j}$ is easily seen to fulfill the requirements of (c).

$(\mathrm{c}) \Rightarrow(\mathrm{d})$ : Every $E \in \mathscr{C}_{r}$ can be identified with $R^{(J)} / L$ for some $L \subset R^{(J)}$. If $\varepsilon: M^{I} \rightarrow M^{I} / M^{(I)}$ denotes the canonical quotient map, then $\varepsilon_{*}:$ Hom $\left(R^{(J)}, M^{I}\right) \rightarrow \operatorname{Hom}\left(R^{(J)}, M^{I} / M^{(I)}\right)$ is onto since $R^{(J)}$ is free. Lemma 2.5 now shows that $\varepsilon_{*}: \operatorname{Hom}\left(E, M^{I}\right) \rightarrow \operatorname{Hom}\left(E, M^{I} / M^{(I)}\right)$ is onto.

$(\mathrm{d}) \Rightarrow(\mathrm{e})$ is trivial.

To complete the proof of Proposition 2.6 we have only to show that $(\mathrm{e}) \Rightarrow$ (b) when $r$ is a finite cardinal. If possible let $\underline{x}^{(n)} \in M^{J}$, $\underline{\lambda}^{(n)} \in R^{(J)}$ satisfy (i) and (ii) of (b). Let $\underline{x}^{(n)}=\left(x_{j}^{(n)}\right), \underline{\lambda}^{(n)}=\left(\lambda_{j}^{(n)}\right)$. Let $\underline{y}^{j}=\left(y_{n}^{j}\right)_{n \geqq 1} \in M^{N}$ be given by $y_{n}^{j}=x_{j}^{(n)}$. Let $f: R^{(J)} \rightarrow M^{N}$ be the map carrying $\underline{e}^{j}$ onto $\underline{y}^{j}$. Let $L$ be the submodule of $R^{(J)}$ generated by $\underline{\lambda}^{(n)}, n \geqq 1$. Then $R^{(J)} / L \in \mathscr{C}_{r}^{\prime}$. The $m$ th coordinate of $f\left(\underline{\lambda}^{(n)}\right)$ is $\sum_{j} y_{m}^{j} \lambda_{j}^{(n)}$. But $\sum_{j} y_{m}^{j} \lambda_{j}^{(n)}=\sum_{j} x_{j}^{(m)} \lambda_{j}^{(n)}=\left\langle\underline{\boldsymbol{x}}^{(m)}, \underline{\lambda}^{(n)}\right\rangle$. Since $\left\langle\underline{\boldsymbol{x}}^{(m)}, \underline{\boldsymbol{\lambda}}^{(n)}\right\rangle=0$ for $m>n$, we get $f\left(\underline{\lambda}^{(n)}\right) \in M^{(N)}$. Hence $f(L) \subset M^{(N)}$. Let $\varepsilon: M^{N} \rightarrow$ $M^{N} / M^{(N)}$ denote the quotient map. By (e) the map $\varepsilon_{*}: \operatorname{Hom}\left(R^{(J)} / L\right.$, $\left.M^{N}\right) \rightarrow \operatorname{Hom}\left(R^{(J)} / L, M^{N} / M^{(N)}\right)$ is onto. Hence by Lemma 2.4 there exists a map $g: R^{(J)} \rightarrow M^{(N)}$ satisfying $g / L=f / L$. Now let $g\left(\underline{e}^{j}\right)=$ $\left(u_{n}^{j}\right)_{n \geq 1}$. From $f\left(\underline{\lambda}^{(n)}\right)=g\left(\underline{\lambda}^{(n)}\right)$ we immediately get $\sum_{j} y_{m}^{j} \lambda_{j}^{(n)}=\sum_{j} u_{m}^{j} \lambda_{j}^{(n)}$ for all $m$. In particular, $\sum_{j} u_{n}^{j} \lambda_{j}^{(n)}=\sum_{j} y_{n}^{j} \lambda_{j}^{(n)}=\sum_{j} x_{j}^{(n)} \lambda_{j}^{(n)} \neq 0$ by (i) 
of (b). Hence for any $n \geqq 1$, there exists at least one $j \in J$ such that $u_{n}^{j} \neq 0$. Since $J$ is a finite set and $n$ varies over the infinite set $N$, it follows that there exists at least one $j \in J$ with $u_{n}^{j} \neq 0$ for infinitely many $n$. This contradicts the fact that $\left(u_{n}^{j}\right)$ is in $M^{(N)}$.

This completes the proof of Proposition 2.6. Actually the proof is patterned after the proof of Lenzing [Proposition 1 of [6]].

It follows from this proposition that $A_{r} \Leftrightarrow A_{(r)}$ for any cardinal $r$. However, we will continue to use both the notations. Another consequence of the proposition is the equivalence of $A_{k}$ and $P_{k}$. In general, we only have $A_{r} \Rightarrow P_{r}$.

Proposition 2.7. Consider the following conditions:

(a) $M$ has property $D_{r}$.

(b) There do not exist sequences $\left\{\underline{x}^{(n)}\right\}$ in $M^{J}$ and $\left\{\underline{\lambda}^{(n)}\right\}$ is $R^{(J)}$ satisfying

$\left.\begin{array}{l}\text { (i) }\left\langle\underline{x}^{(n)}, \underline{\lambda}^{(n)}\right\rangle \neq 0 \\ \text { (ii) }\left\langle\underline{\boldsymbol{x}}^{(n)}, \underline{\lambda}^{(m)}\right\rangle=0\end{array}\right\}$ for $m>n \geqq 1$.

(a') $M$ has property $D_{(r)}$.

(b') Same as (b) with $M^{(J)}$ replacing $M^{J}$.

Then we have $(\mathrm{a}) \Leftrightarrow(\mathrm{b}),\left(\mathrm{a}^{\prime}\right) \Leftrightarrow\left(\mathrm{b}^{\prime}\right)$ and $(\mathrm{b}) \Rightarrow\left(\mathrm{b}^{\prime}\right)$ (thus $(\mathrm{a}) \Rightarrow\left(\mathrm{b}^{\prime}\right)$ ).

Proof. The equivalences $(a) \Leftrightarrow(b),\left(a^{\prime}\right) \Leftrightarrow\left(b^{\prime}\right)$ follow from Proposition 1.2. The implication $(\mathrm{b}) \Leftrightarrow\left(\mathrm{b}^{\prime}\right)$ is trivial since $M^{(J)} \subset M^{J}$.

CoRollary 2.8. Let $G_{r}$ denote any one of the properties $A_{r}$, $D_{r}, D_{(r)}$.

(i) Let $N$ be a submodule of $M$. If $M$ has $G_{r}$ so does $N$.

(ii) If $M_{1}, M_{2}$ are any two modules having $G_{r}$ so does $M_{1} \oplus M_{2}$.

EXAMPLE 2.9. In mod- $Z$ the sequence $0 \rightarrow Q^{(I)} \rightarrow Q^{I} \rightarrow Q^{I} / Q^{(I)} \rightarrow 0$ is split exact and hence pure-exact with respect to any family of modules. In particular, $Q$ has property $P_{r}$ for every cardinal $r$. However, we will show that $Q$ does not have $A_{r_{0}}$. Let $\underline{\boldsymbol{x}}^{(k)} \in Q^{N}$, $\underline{\lambda}^{(k)} \in \boldsymbol{Z}^{(N)}$ be defined by $\underline{\lambda}^{(k)}=\left(\lambda_{n}^{(k)}\right)_{n \geqq 1}$ where

$$
\lambda_{n}^{(k)}=\left\{\begin{array}{ll}
(-1)^{n+1}(n+1) & \text { for } n \leqq 2 k \\
0 & \text { for } n>2 k
\end{array}\right\}
$$

for all $k \geqq 1 ; \underline{x}^{(1)}=(1 / n+3)_{n \geqq 1}$ and $\underline{x}^{(k)}=\left(x_{n}^{(k)}\right)$ where

$$
x_{n}^{(k)}=\left\{\begin{array}{l}
\frac{1}{n+1} \text { for } n \leqq 2 k-2 \\
\frac{1}{n+3} \text { for } n \geqq 2 k-1
\end{array}\right\}
$$


for $k \geqq 2$. Then $\left\langle\underline{x}^{(l)}, \underline{\lambda}^{(k)}\right\rangle=0$ for all $l>k \geqq 1$ and $\left\langle\underline{x}^{(k)}, \underline{\lambda}^{(k)}\right\rangle \neq 0$ for all $k \geqq 1$. Hence by Proposition 2.6, $Q$ does not have $A_{r_{0}}$.

EXAMPLe 2.10. Since $A_{k} \Leftrightarrow P_{k}$ it follows from Corollary 2.8 that $P_{k}$ is a hereditary property. However, for an infinite cardinal $r, P_{r}$ is not hereditary. In mod- $Z, Q$ has $P_{r}$ for all $r$. But $Z$ does not have $P_{\mathscr{S}_{0}}$. Let $p$ be any prime and $\underline{\boldsymbol{u}}^{(k)} \in \boldsymbol{Z}^{N}$ be defined by $u_{n}^{(k)}=$ $\begin{cases}0 & \text { if } n \leqq k-1 . \text { Let } \varepsilon: \boldsymbol{Z}^{N} \rightarrow \boldsymbol{Z}^{N} / \boldsymbol{Z}^{(N)} \text { denote the quotient map. If } \\ p^{n-k} & \text { if } n \geqq k\end{cases}$ $v^{(k)}=\varepsilon\left(\underline{u}^{(k)}\right)$ then $p v^{(k+1)}=v^{(k)}$ for all $k$. Let $\Gamma$ denote the subgroup of $Q$ consisting of elements of the form $m / p^{r}$ with $m \in \boldsymbol{Z}, r \geqq 1$. There exists a unique homomorphism $f: \Gamma \rightarrow Z^{N} / Z^{(N)}$ satisfying $f(1)=$ $v^{(1)}, f\left(1 / p^{k}\right)=v^{(k+1)}$. However, $\operatorname{Hom}\left(\Gamma, Z^{N}\right)=0$.

REMARK 2.11. Suppose $R$ is a commutative ring. Using Proposition 1.3, it is easily seen that any artinian (resp. noetherian) module $M$ has property $A_{k}$ (resp. $D_{k}$ ) for all $k \geqq 1$. In particular, in mod- $\boldsymbol{Z}$, the modules $M_{n}=\boldsymbol{Z} / p^{n} \boldsymbol{Z}$ have both $A_{k}$ and $D_{k}$. If $M=$ $\bigoplus_{n \geqq 1} M_{n}$, then $p \boldsymbol{Z} \supset p^{2} \boldsymbol{Z} \supset \cdots$ is a strict descending chain in $\mathscr{F}_{1}(M)$. Thus $M$ does not have $D_{1}$.

REMARK 2.12. Let $R=\Pi_{j \in J} K_{j}$ be the direct product of infinitely many fields. Then each $K_{j}$ as an $R$-module has property $A_{k}$ for all $k$. However, $M=\bigoplus_{j \in J} K_{j}$ does not have property $A_{1}$.

Remarks 2.11 and 2.12 show that 2.8(ii) cannot be generalized to infinite direct sums (hence by 2.8(i) to infinite direct products of modules as well). The following result is essentially due to $\mathrm{C}$. Faith [4].

Proposition 2.13. The following conditions on a ring $R$ are equivalent.

(a) Every injective module has property $A_{1}$.

(b) Every module has property $A_{1}$.

(c) $R$ is noetherian.

(d) Every module has property $A_{k}$ for all $k \geqq 1$.

Proof. $\quad(a) \Rightarrow$ (b) is immediate from 2.8(i), since every module is a submodule of an injective module.

(b) $\Rightarrow(\mathrm{c})$ : Let $\mathscr{H}$ denote the family of all (right) ideals of $R$ and $M=\bigoplus_{L \in \dddot{*}} R / L$. Then $\mathscr{F}_{1}(M)=\mathscr{H}$.

$(\mathrm{c}) \Rightarrow(\mathrm{d})$ : When $R$ is noetherian, $R^{k}$ is noetherian. Hence $\mathscr{F}_{k}(M)$ has the a.c.c. for any $M \in \bmod -R$.

$(\mathrm{d}) \Rightarrow(\mathrm{a})$ is trivial.

Proposition 2.14. The following conditions on a ring $R$ are 
equivalent.

(a) Every injective module has property $D_{1}$.

(b) Every module has property $D_{1}$.

(c) $R$ is artinian.

(d) Every module has property $D_{k}$ for all $k \geqq 1$.

The proof is similar to that of Proposition 2.13 and hence omitted.

REMARK 2.15. Let $M$ be a module having the property $A_{1}$. Then by Faith's theorem [4], if $M$ is injective, then it is $\Sigma$-injective (namely, $M^{(I)}$ is injective for every set $I$ ). In particular, $M^{(I)}$ is a direct summand of $M^{I}$ for any set $I$. However, this property does not characterize injective modules among modules having $A_{1}$. Let $R$ be a left artinian ring which is not self-injective. Then by Proposition 3 of Lenzing [6] it follows that $R^{(I)}$ is a direct summand of $R^{I}$ for all $I$. But $R$ is not injective.

3. Behavior under base extension. Let $R$ be a subring of $S, M \in \bmod -R$ and $M^{\prime}=M \otimes_{R} S \in \bmod -S . \quad J$ denotes a set with $|J|=r$.

TheOREM 3.1. Suppose $\operatorname{Tor}_{1}^{R}(M, S / R)=0$. If $M^{\prime}$ has property $A_{(r)}$ (resp. $\left.D_{(r)}\right)$, then so does $M$.

Proof. We will prove this for $A_{(r)}$ (the proof for $D_{(r)}$ is similar and hence omitted). If $M$ does not have $A_{(r)}$, there exist sequences $\underline{\boldsymbol{x}}^{(n)} \in M^{(J)}$ and $\underline{\boldsymbol{\lambda}}^{(n)} \in R^{(J)}$ such that $\left\langle\underline{\boldsymbol{x}}^{(n)}, \underline{\lambda}^{(n)}\right\rangle \neq 0$ and $\left\langle\underline{\boldsymbol{x}}^{(m)}, \underline{\lambda}^{(n)}\right\rangle=0$ for $m>n \geqq 1$. Let $\underline{y}^{(n)}=x^{(n)} \otimes 1 \in M^{(J)} \otimes S=M^{\prime^{(J)}}$. Regarding $\underline{\lambda}^{(n)}$ as elements of $S^{(j)}$ we have

$$
\left\langle\underline{y}^{(m)}, \underline{\lambda}^{(n)}\right\rangle=\left\langle\underline{\boldsymbol{x}}^{(m)} \otimes 1, \underline{\lambda}^{(n)}\right\rangle=\left\langle\underline{\boldsymbol{x}}^{(m)}, \underline{\boldsymbol{\lambda}}^{(n)}\right\rangle \otimes 1 \in M^{\prime} .
$$

When $\operatorname{Tor}_{1}^{R}(M, S / R)=0$, the map $u \mapsto u \otimes 1$ of $M$ in $M^{\prime}$ is injective. Hence $\left\langle\underline{y}^{(n)}, \underline{\lambda}^{(n)}\right\rangle \neq 0$ and $\left\langle\underline{y}^{(m)}, \underline{\lambda}^{(n)}\right\rangle=0$ for $m>n \geqq 1$. This means $M^{\prime}$ does not have $A_{(r)}$.

REMARK 3.2. In general $M^{J} \otimes S \neq(M \otimes S)^{J}$. If $S$ is finitely presented as a (left) $R$-module we can identify $M^{J} \otimes S$ with $(M \otimes S)^{J}$. In this case the analogue of Theorem 3.1 is valid for the property $D_{r}$ as well.

Proposition 3.3. Suppose $R$ is a subring of a noetherian (resp. artinian) ring $S$. Then any $M \in \bmod -R$ with $\operatorname{Tor}_{1}^{R}(M, S / R)=0$ has property $A_{k}$ (resp. $D_{k}$ ).

Proof. Immediate consequence of Propositions 2.13,, 2.14 and 
Theorem 3.1.

CoROLlaRY 3.4. Let $R$ be a subring of a ring $S$ and let $S / R$ be flat as a left $R$-module (equivalently, let $S$ be a faithfully flat left $R$-module). If $S$ is right noetherian (artinian), then $R$ is right noetherian (artinian).

This corollary is actually proved in $\S 3$, No. 5, Chap. I, of [2] by different methods.

Proposition 3.5. Let $R$ be a von Neumann regular ring. Then $R$ is semi-simple $\Leftrightarrow R$ has $A_{1}$.

Proof. Immediate consequence of Proposition 2.6 and the wellknown fact that a regular ring $R$ is semi-simple $\Leftrightarrow$ there does not exist an infinite family of orthogonal idempotents.

4. Behavior of $\operatorname{Hom}_{R}(S, M)$. Unless otherwise mentioned we consider the following situation. $R$ is a subring of $S$ and $i: R \rightarrow S$ denotes the inclusion. We assume that there exists an augmentation $\varepsilon: S \rightarrow R$ (namely, a ring homomorphism satisfying $\varepsilon \circ i=I d_{R}$ ). Let $K=\operatorname{ker} \varepsilon$ be the augmentation ideal in $S$. Then as an $R$-module we have $S=R \oplus K$. Moreover, $S K=K=K S$. In particular, $R K \subset K$. In what follows $J$ denotes a set with $|J|=r$.

LEMMA 4.1. Let $\left\{\underline{u}^{(\mu)}\right\}$ be any family of elements of $R^{(J)}$. Let $N$ be the $R$-submodule of $R^{(J)}$ generated by $\left\{\underline{u}^{(\mu)}\right\}$ and $V$ the $S$-submodule of $S^{(J)}$ generated by $\left\{\underline{u}^{(\mu)}\right\}$.

Then $V \cap R^{(J)}=N$.

Proof. Clearly $V \cap R^{(J)} \supset N$. Let $l=\Sigma \underline{u}^{(\mu)} s_{\mu} \in V \cap R^{(J)}$ with $s_{\mu} \in S$ (and $s_{\mu}=0$ for almost all $\mu$ ). Let $s_{\mu}=r_{\mu}+t_{\mu}$ with $r_{\mu} \in R$, $t_{\mu} \in K$. If $\underline{\boldsymbol{u}}^{(\mu)}=\left(u_{j}^{(\mu)}\right)_{j \in J}$ we have $\underline{\boldsymbol{u}}^{(\mu)} \cdot t_{\mu}=\left(u_{j}^{(\mu)} t_{\mu}\right)_{j \in J}$. Since $R K \subset K$, it follows that $u_{j}^{(\mu)} t_{\mu} \in K$ for each $j \in J$. Hence $\underline{u}^{(\mu)} t_{\mu} \in K^{(J)}$. As an $R$-module we have $S^{(J)}=R^{(J)} \oplus K^{(J)}$ and the element $l$ of $R^{(J)}$ has the representation $\left(\sum_{\mu} \underline{\underline{u}}^{(\mu)} r_{\mu}\right)+\left(\sum_{\mu} \underline{\underline{u}}^{(\mu)} t_{\mu}\right)$ with $\sum_{\mu} \underline{\underline{u}}^{(\mu)} r_{\mu} \in R^{(J)}$ and $\sum_{\mu} \underline{u}^{(\mu)} t_{\mu} \in K^{(J)}$. It follows that $l=\sum_{\mu} \underline{u}^{(\mu)} r_{\mu}$. In other words, $l \in N$. Thus $V \cap R^{(J)} \subset N$.

Let $\Gamma_{r}$ (resp. $\Gamma_{r}^{\prime}$ ) dedote the class of $R$-submodules of $S^{(J)}$ of the form $R^{(J)}+V$ where $V$ is an $S$-submodule of $S^{(J)}$ generated by finitely many (resp. countably many) element of $R^{(J)}$.

TheOREM 4.2. Suppose $M \in \bmod -R$ satisfies $\operatorname{Ext}_{R}^{1}\left(S^{(J)} / L, M\right)=0$ for any $L \in \Gamma_{r}$ (resp. $\left.\Gamma_{r}^{\prime}\right)$. Let $M^{\prime}=\operatorname{Hom}_{R}(S, M)$. If $M^{\prime}$ has $A_{n}$ 
(resp. $D_{r}$ ), then $M$ has $A_{r}$ (resp. $D_{r}$ ).

Proof. We prove the theorem only for $A_{r}$. The proof for $D_{r}$ is similar and hence omitted. Suppose $M$ does not have $A_{r}$. Then there exist $\underline{x}^{(n)} \in M^{J}, \underline{\lambda}^{(n)} \in R^{(J)}$ with $\left\langle\underline{x}^{(n)}, \underline{\lambda}^{(n)}\right\rangle \neq 0$ and $\left\langle\underline{x}^{(m)}, \underline{\lambda}^{(n)}\right\rangle=0$ for $m>n \geqq 1$. We identify $M^{J}$ with $\operatorname{Hom}\left(R^{(J)}, M\right)$. An element $\underline{x} \in M^{J}$ corresponds to the homomorphism $\alpha_{\underline{x}}: R^{(J)} \rightarrow M$ given by $\alpha_{x}(\underline{\lambda})=$ $\langle\underline{x}, \underline{\lambda}\rangle$. For $n \geqq 2$ let $V_{n}$ be the $S$-submodule of $S^{(J)}$ generated by $\underline{\lambda}^{(1)}, \cdots, \underline{\lambda}^{(n-1)}$ and $L_{n}$ the $R$-submodule of $R^{(J)}$ generated by $\underline{\lambda}^{(1)}, \cdots$, $\underline{\lambda}^{(n-1)}$. From Lemma 4.1 we get $L_{n}=V_{n} \cap R^{(J)}$. Denote the map $\alpha_{\underline{x}}(n): R^{(J)} \rightarrow M$ by $f^{(n)}$. Then $f^{(n)}\left(L_{n}\right)=0$. If $g^{(n)}: R^{(J)}+V_{n} \rightarrow M$ is defined by $g^{(n)}(\underline{u}+\underline{v})=f^{(n)}(\underline{u})$ for any $\underline{u} \in R^{(J)}, \underline{v} \in V_{n}$, then using the facts that $V_{n} \cap R^{(J)}=L_{n}$ and that $f^{(n)}\left(L_{n}\right)=0$, it can easily be shown that $g^{(n)}$ is a well-defined $R$-homomorphism. Clearly $R^{(J)}+$ $V_{n} \in \Gamma_{r}$. By assumption, $\operatorname{Ext}_{R}^{1}\left(S^{(J)} / R^{(J)}+V_{n}, M\right)=0$. It follows that $g^{(n)}$ can be extended to an $R$-homomorphism $h^{(n)}: S^{(J)} \rightarrow M$. Under the usual identification of $\operatorname{Hom}_{R}\left(S^{(J)}, M\right)$ with $\operatorname{Hom}_{R}(S, M)^{J}=$ $M^{\prime J}$ let $h^{(n)}$ correspond to the element $\underline{h}^{(n)}=\left(h_{j}^{(n)}\right) \in M^{\prime J}$ with $h_{j}^{(n)} \in M^{\prime}$. Then the element $\left\langle\underline{h}^{(n)}, \underline{\lambda}^{(i)}\right\rangle \in M^{\prime}=\operatorname{Hom}_{R}(S, M)$ satisfies $\left\langle\underline{h}^{(n)}, \underline{\lambda}^{(i)}\right\rangle(s)=$ $\sum_{j} h_{j}^{(n)}\left(\lambda_{j}^{(i)} s\right) \in M$ for every $s \in S$. But $\left\langle\underline{h}^{(n)}, \underline{\lambda}^{(i)} s\right\rangle(1)=\sum_{j} \bar{h}_{j}^{(n)}\left(\lambda_{j}^{(i)} s\right)$. Since $\underline{h}^{(n)} / V_{n}=0$, it follows that $\left\langle\underline{h}^{(n)}, \underline{\lambda}^{(i)} s\right\rangle=0$ for $1 \leqq i \leqq n-1$. In other words, $\left\langle\underline{h}^{(n)}, \underline{\lambda}^{(i)}\right\rangle(s)=0 \quad \forall s \in S$ whenever $1 \leqq i \leqq n-1$. Hence $\left\langle\underline{h}^{(n)}, \underline{\lambda}^{(i)}\right\rangle=0$ in $M^{\prime}$ for $1 \leqq i \leqq n-1$. Also $\left\langle\underline{h}^{(n)}, \underline{\lambda}^{(n)}\right\rangle(1)=$ $\left.f^{(n)} \underline{\lambda}^{(n)}\right)=\left\langle\underline{x}^{(n)}, \underline{\lambda}^{(n)}\right\rangle \neq 0$. Thus $M^{\prime}$ does not have $A_{r}$.

Let $M$ be an injective module in mod-R. Then $M^{\prime}=\operatorname{Hom}_{R}(S, M)$ is injective in mod-S [Proposition 1.4, 1 , Chap. VI, [3]].

COROLLARY 4.3. With the same conventions as at the beginning of this section, let $M \in \bmod -R$ be injective. If $M^{\prime}$ is $\Sigma$-injective in mod-S, then so is $M$ in mod-R.

Proof. This is because an injective module is $\Sigma$-injective if and only if it has $A_{1}$.

REMARK 4.4. Theorem 4.2 and Corollary 4.3 are not valid for an arbitrary ring $S$ containing $R$ as a subring. For instance, let $R$ be a non-noetherian integral domain and $S$ its quotient field. Since $R$ is non-noetherian, there exists an injective module $M \in \bmod -R$ which is not $\Sigma$-injective. However, since $S$ is a field, $M^{\prime}=\operatorname{Hom}_{R}(S, M)$ is $\Sigma$-injective in mod-S.

REMARK 4.5. Let $R$ be any ring and $\mathfrak{A}$ a two-sided ideal of $R$. Let $f: R \rightarrow R / \mathfrak{A}$ be the canonical quotient map. Let $E \in \bmod -R$ and $E^{\prime}=\{x \in E \mid x \mathfrak{A}=0\} \cong \operatorname{Hom}_{R}(R / \mathfrak{A}, E) \in \bmod -R / \mathfrak{A}$. Suppose $E^{\prime}$ does not 
have $A_{1}$ in $\bmod -R / \mathfrak{A}$. Then there exist $x^{(n)} \in E^{\prime}$ and $\lambda^{(n)} \in R$ such that $x^{(n)} f\left(\lambda^{(n)}\right) \neq 0$ and $x^{(m)} f\left(\lambda^{(n)}\right)=0$ for $m>n \geqq 1$. But $x^{(n)} \lambda^{(i)}=x^{(n)} f\left(\lambda^{(i)}\right)$. Thus $x^{(n)} \lambda^{(n)} \neq 0$ and $x^{(m)} \lambda^{(n)}=0$ for $m>n \geqq 1$. Since $x^{(n)} \in E$ it follows that $E$ does not have $A_{1}$. Now if $E$ is $R$-injective, then $E^{\prime}$ is $R / \mathfrak{Q}$-injective. By the argument above if $E$ is $\Sigma$-injective, then so is $E^{\prime}$ in $\bmod -R / \mathfrak{A}$. Since $E^{\prime(I)}=\left\{x \in E^{(I)} \mid x \mathfrak{A}=0\right\}$ this last fact can also be seen directly.

5. Arbitrary family $M_{\alpha}$. Let $\left\{M_{\alpha}\right\}_{\alpha \in I}$ be any family of modules. For any $k$-tuple $\left(\underline{x}^{(1)}, \cdots, \underline{x}^{(k)}\right)$ with $\underline{x}^{(i)} \in \Pi M_{\alpha}$ let $T_{\left(\underline{x}^{(1)}, \ldots, \underline{x}^{(k))}\right.}=$ $\left\{\left(\lambda^{(1)}, \cdots, \lambda^{(k)}\right) \in R^{k} \mid \sum_{i=1}^{k} \underline{x}^{(i)} \lambda^{(i)} \in \bigoplus M_{\alpha}\right\}$.

Definition 5.1. A $k$-tuple $\left(\underline{x}^{(1)}, \cdots, \underline{x}^{(k)}\right)$ with $\underline{x}^{(i)} \in \Pi M_{\alpha}$ is called "special" if there exists a finite subset $F$ of $I$ such that $\sum_{i=1}^{k} x_{\alpha}^{(i)} \lambda^{(i)}=0$ for all $\alpha \in I-F$ and $\left(\lambda^{(1)}, \cdots, \lambda^{(k)}\right) \in T_{\left(\underline{x}^{(1)}, \ldots, \underline{x}^{(k)}\right)}$. Here $\underline{x}^{(i)}=\left(x_{\alpha}^{(i)}\right)_{\alpha \in I}$. For any integer $k \geqq 1$ the following result is the analogue of Proposition 2.6. Its proof is practically similar to that of Proposition 2.6 and hence is omitted.

Proposition 5.2. Let $\left\{M_{\alpha}\right\}_{\alpha \in I}$ be any family of $R$-modules and $k$ an integer $\geqq 1$. Then the following statements are equivalent.

(a) Each $k$-tuple $\left(\underline{x}^{(1)}, \cdots, \underline{x}^{(k)}\right)$ of elements from $\Pi M_{\alpha}$ is special.

(b) For every countable infinite subset $X$ of I and any enumeration of $X$, there do not exist families of elements $\left(x_{\mu}^{(1)}\right)_{\mu \in X}, \cdots,\left(x_{\mu}^{(k)}\right)_{\mu \in X}$ in $\prod_{u \in X} M_{\mu}$ and $\left(\lambda_{\mu}^{(1)}\right)_{\mu \in X}, \cdots,\left(\lambda_{\mu}^{(k)}\right)_{\mu \in X}$ is $R^{(X)}$ satisfying

(i) $\sum_{i=1}^{k} x_{\mu}^{(i)} \lambda_{\mu l}^{(i)} \neq 0$ for all $\mu \in X$ and

(ii) $\sum_{i=1}^{k} x_{\nu}^{(i)} \lambda_{\mu}^{(i)}=0$ for $\nu>\mu$ in $X$.

(c) The sequence $0 \rightarrow \bigoplus M_{\alpha} \rightarrow \Pi M_{\alpha} \rightarrow \Pi M_{\alpha} / \oplus M_{\alpha} \rightarrow 0$ is pureexact with respect to $\mathscr{C}_{k}$.

(d) For every countable subset $X$ of $I$ the sequence $0 \rightarrow \bigoplus_{\alpha \in X} M_{\alpha} \rightarrow$ $\prod_{\alpha \in X} M_{\alpha} \rightarrow \prod_{\alpha \in X} M_{\alpha} / \bigoplus_{\alpha \in X} M_{\alpha} \rightarrow 0$ is pure-exact with respect to $\mathscr{C}_{k}^{\prime}$.

(e) For any $L \subset R^{(k)}$ and $f: R^{(k)} \rightarrow \Pi M_{\alpha}$ with $f(L) \subset \bigoplus M_{\alpha}$ there exists a $g: R^{(k)} \rightarrow \bigoplus M_{\alpha}$ satisfying $g / L=f / L$.

\section{REFERENCES}

1. F. W. Anderson and K. R. Fuller, Rings and Categories of Modules, SpringerVerlag, New York, 1974.

2. N. Bourbaki, Algèbre Commutative, Chap. 1 and 2, Hermann, Paris, 1961.

3. H. Cartan and S. Eilenberg, Homological Algebra, Princeton University Press, Princeton, 1956.

4. C. Faith, Rings with ascending chain condition on annihilators, Nagoya Math. J., 27 (1966), 179-191.

5. D. J. Fieldhouse, Pure theories, Math. Ann., 184 (1969), 1-18.

6. H. Lenzing, Direct sums of projective modules as direct summands of their direct product, Comm. in Algebra, 4 (1976), 681-691. 
7. B. Sarath and K. Varadarajan, Injectivity of direct sums, Comm. in Algebra, $\mathbf{1}$ (1974), 517-530.

Received March 1, 1977. Research done while the second author was spending part of his sabbatical leave at the Tata Institute of Fundamental Research, Bombay. The hospitality extended to him by the T.I.F.R. and support from NRC grant A8225 are gratefully acknowledged.

Tata Institute of Fundamental Research

Homi BhabHa ROAD, Bombay 400 005, India

AND

The University of Calgary

Calgary, Alberta, Canada T2N 1N4 



\title{
PACIFIC JOURNAL OF MATHEMATICS
}

\author{
EDITORS
}

RICHARD ARENS (Managing Editor)

University of California

Los Angeles, California 90024

C. W. CurTis

University of Oregon

Eugene, OR 97403

C. C. MOORE

University of California

Berkeley, CA 94720
J. DUgundJI

Department of Mathematics

University of Southern Californı

Los Angeles, California 90007

R. Finn and J. Milgram

Stanford University

Stanford, California 94305

\section{ASSOCIATE EDITORS}

E. F. BECKENBACH

\section{SUPPORTING INSTITUTIONS}

\author{
UNIVERSITY OF BRITISH COLUMBIA \\ CALIFORNIA INSTITUTE OF TECHNOLOGY \\ UNIVERSITY OF CALIFORNIA \\ MONTANA STATE UNIVERSITY \\ UNIVERSITY OF NEVADA, RENO \\ NEW MEXICO STATE UNIVERSITY \\ OREGON STATE UNIVERSITY \\ UNIVERSITY OF OREGON \\ OSAKA UNIVERSITY
}

UNIVERSITY OF SOUTHERN CALIFORNIA

STANFORD UNIVERSITY

UNIVERSITY OF TOKYO

UNIVERSITY OF UTAH

WASHINGTON STATE UNIVERSITY

UNIVERSITY OF WASHINGTON
AMERICAN MATHEMATICAL SOCIETY
NAVAL WEAPONS CENTER 


\section{Pacific Journal of Mathematics \\ Vol. 72 , No. 1 \\ January, 1977}

Kazuo Anzai and Shiro Ishikawa, On common fixed points for several

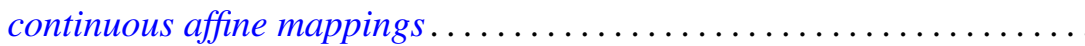

Bruce Alan Barnes, When is a representation of a Banach $*$-algebra

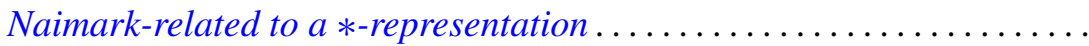

Richard Dowell Byrd, Justin Thomas Lloyd, Franklin D. Pedersen and

James Wilson Stepp, Automorphisms of the semigroup of finite

complexes of a periodic locally cyclic group ...................

Donald S. Coram and Paul Frazier Duvall, Jr., Approximate fibrations and a

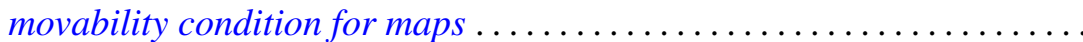

Kenneth R. Davidson and Che-Kao Fong, An operator algebra which is not

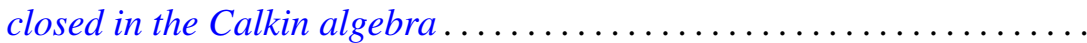

Garret J. Etgen and James Pawlowski, A comparison theorem and oscillation criteria for second order differential systems .............

Philip Palmer Green, $C^{*}$-algebras of transformation groups with smooth

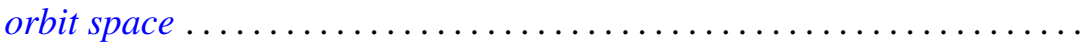

Charles Allen Jones and Charles Dwight Lahr, Weak and norm approximate

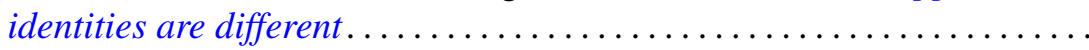

G. K. Kalisch, On integral representations of piecewise holomorphic

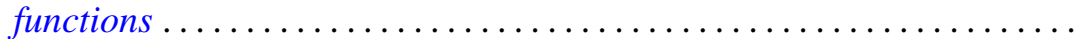

Y. Kodama, On product of shape and a question of Sher

Heinz K. Langer and B. Textorius, On generalized resolvents and

$Q$-functions of symmetric linear relations (subspaces) in Hilbert

space ...................................

Albert Edward Livingston, On the integral means of univalent, meromorphic functions

Wallace Smith Martindale, III and Susan Montgomery, Fixed elements of

Jordan automorphisms of associative rings ..........

R. Kent Nagle, Monotonicity and alternative methods for nonlinear boundary value problems ........................

Richard John O'Malley, Approximately differentiable functions: the $r$ topology.

Mangesh Bhalchandra Rege and Kalathoor Varadarajan, Chain conditions

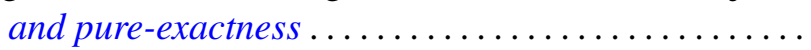

Christine Ann Shannon, The second dual of $C(X)$. .

Sin-ei Takahasi, A characterization for compact central double centralizers of $C^{*}$-algebras .

Theresa Phillips Vaughan, A note on the Jacobi-Perron algorithm. . .

Arthur Anthony Yanushka, A characterization of $\operatorname{PSp}(2 m, q)$ and

$\mathrm{P} \Omega(2 m+1, q)$ as rank 3 permutation groups ......... 